\title{
Significance of connective tissue diseases features in pulmonary fibrosis
}

\author{
Vincent Cottin ${ }^{1,2}$
}

\begin{abstract}
Affiliations: ${ }^{1}$ Hospices Civils de Lyon, Hôpital Louis Pradel, Service de pneumologie - Centre de référence national des maladies pulmonaires rares et Centre de compétences de l'hypertension artérielle pulmonaire, Lyon, and ${ }^{2}$ Université de Lyon, Université Claude Bernard Lyon 1, INRA, UMR754 INRA-Vetagrosup EPHE IFR 128, Lyon, France.

Correspondence: V. Cottin, National Reference Center for Rare Pulmonary Diseases, Competence Center for Pulmonary Hypertension, Dept of Respiratory Diseases - Louis Pradel Hospital, Claude Bernard Lyon 1 University, 28 Avenue du Doyen Lepine, 69677 Lyon, France. E-mail: vincent.cottinachu-lyon.fr
\end{abstract}

ABSTRACT Interstitial lung disease (ILD) can occur in any of the connective tissue diseases (CTD) with varying frequency and severity, and an overall long-term prognosis that is less severe than that of idiopathic pulmonary fibrosis (IPF). Because ILD may be the presenting manifestation of CTD and/or the dominant manifestation of CTD, clinical extra-thoracic manifestations should be systematically considered in the diagnostic approach of ILD. When present, autoantibodies strongly contribute to the recognition and classification of the CTD.

Patients with clinical extrathoracic manifestations of CTD and/or autoantibodies (especially with a high titer and/or the antibody is considered "highly specific" of an autoimmune condition), but who do not fit with established international CTD criteria may be called undifferentiated CTD or "lung-dominant CTD". Although it remains to be determined which combination of symptoms and serologic tests best identify the subset of patients with clinically relevant CTD features, available evidence suggests that such patients may have distinct clinical and imaging presentation and may portend a distinct clinical course. However, autoantibodies alone when present in IPF patients do not seem to impact prognosis or management. Referral to a rheumatologist and multidisciplinary discussion may contribute to management of patients with undifferentiated CTD.

○ @ERSpublications

ILD should be classified as undifferentiated connective tissue disease if at least one auto-antibody and at least one extra-thoracic feature is present http://ow.ly/mMJLW

\section{Introduction}

Interstitial lung disease (ILD) can occur in any connective tissue disease (CTD), also referred to as collagen vascular diseases, with varying frequency and severity $[1,2]$. The long-term prognosis of patients with CTDILD has recently been shown to be less severe than that of idiopathic pulmonary fibrosis (IPF) [3, 4]. However, a subset of patients present with symptoms that belong to the spectrum of clinical manifestations of CTDs and/or biological autoimmune features, but do not fulfil diagnostic criteria for a given autoimmune disease. Here, we briefly review the clinical relevance of this condition, which has been tentatively given various terminologies; the most frequently used being that of undifferenciated CTD (UCTD). This review is a summary of a lecture given during the 5th international meeting on Pulmonary Rare Diseases and Orphan Drugs, held in Milan, Italy.

Received: May 132013 | Accepted: May 282013

Conflict of interest: Disclosures can be found alongside the online version of this article at err.ersjournals.com

Provenance: Publication of this peer-reviewed article was supported by the World Scleroderma Foundation, Switzerland (principal sponsor, European Respiratory Review issue 129).

Copyright @ERS 2013 


\section{When should CTD be suspected?}

CTDs are a heterogeneous spectrum of systemic rheumatologic conditions, which, collectively, are characterised by systemic organ manifestations and autoimmune manifestations, e.g. presence of autoantibodies. Respiratory manifestations in a patient with CTD should first lead to the consideration of possible infectious complications, especially in immunocompromised hosts, drug-induced ILD, especially in patients with rheumatoid arthritis receiving non-steroidal anti-inflammatory drugs or newer biologic agents, and complications of the CTD with indirect respiratory consequences (e.g. dyspnoea related to renal heart failure in patients with systemic lupus erythematosus or heart failure in systemic sclerosis) [2].

As ILD may occur in any CTD, clinical manifestations that may be associated with ILD are manifold, and a thorough analysis of clinical history and symptoms is needed. However, some manifestations are frequently encountered and should be searched for as a priority during the diagnostic process of a patient presenting with ILD. Any patient presenting with ILD should be evaluated for the presence of Raynaud's phenomenon (with nailfold capillaroscopy indicated when present), skin manifestations, dryness of eyes and mouth, and joint and muscle symptoms (table 1). Although not exhaustive, focusing on these manifestations is probably the most time-effective for respiratory physicians.

Systemic manifestations have consistently been shown to have a higher prevalence in female patients and individuals aged $<50$ years $[5,6]$. Furthermore, the presence of an underlying CTD is more likely in patients who have a high-resolution computed tomography (HRCT) pattern of nonspecific interstitial pneumonia (NSIP), as opposed to those with a pattern of usual interstitial pneumonia (UIP) [5]. A typical pattern of UIP (e.g. with presence of honeycombing, reticulation with basal and subpleural predominance, and lack of features suggesting an alternative condition) is less frequent in patients with CTD than in those with IPF, even in the setting of histologically proven UIP [7]. In addition, some imaging features may occasionally contribute to suggest the presence of CTD, such as oesophageal dilatation in patients with systemic sclerosis or pneumomediastinum preferentially encountered in patients with idiopathic inflammatory myopathy [8]. Coexistence of emphysema characterising the syndrome of combined pulmonary fibrosis and emphysema is less frequent in patients with CTD than in idiopathic disease [7]; it may especially occur in patients with rheumatoid arthritis, however, other CTDs may be present including systemic sclerosis [9].

Similarly, histopathological features may suggest the possibility of CTD, especially the NSIP pattern, which is the most frequently observed pattern in this setting (with the possible exception of rheumatoid arthritis that is more frequently associated with the UIP pattern) $[3,10-16]$. The histological pattern of UIP is generally nonspecific and indistinguishable according to the aetiological context (e.g. idiopathic, CTD, asbestos exposure, drugs, hypersensitivity pneumonitis). However, as compared to patients with IPF, those with histological UIP in the setting of CTD may have fewer fibroblastic foci, less severe honeycombing, more prominent lymphoid hyperplasia with germinal centres and more extensive plasmatic infiltration, as well as extensive pleuritis and dense perivascular collagen accumulation [2, 7, 17]. Histopathogical features specific of CTD are exceptional and limited to rheumatoid granulomatous inflammation (which can be observed in the pleura or lung nodules of patients with rheumatoid arthritis). A combination of

\section{TABLE 1 Key symptoms to detect underlying connective tissue disease}

\section{Peripheral circulation Skin}

Joints

Muscle

Mouth and eyes
Raynaud's phenomenon

Sclerodactily

Digital ulcerations or scars

Telangiectasia

Violaceous erythematous rash over the interphalangeal joints, knuckles, elbows and knees (Gottron's sign)

Lilaceous rash of the eyelids

Rash of the neck and upper chest and shoulders (heliotrope rash, e.g. photosensitivity) Mechanics' hand

Joint pain or swelling (arthritis, arthralgia)

Morning stiffness

Muscle pain and muscle weakness

Dry mouth and dry eyes (sicca syndrome)

\footnotetext{
\#: this list is not exhaustive.
} 
histopathological patterns may also suggest an underlying CTD (e.g. the combinations of a UIP pattern with prominent lymphocytic inflammation and lymphocytic or follicular bronchiolitis are particularly frequent in patients with rheumatoid arthritis) [1, 18].

\section{Does the diagnosis of established CTD affect prognosis or management?}

Although the relative severity of CTD-ILD and IPF has been a matter of debate for many years, it has been demonstrated recently that patients with IPF have an increased risk of mortality as compared to those with CTD-ILD. This is based on studies conducted in the community and collectively involving different CTDs $[3,4]$, as well as other studies emanating from highly specialised centres and usually focused on one of the CTDs [19]. This observation holds true when considering individual CTDs, and is only partially attributable to the higher frequency of the NSIP pattern in this patient population [3]. However, the survival advantage of CTD-ILD compared to IPF may be less significant in the subset of patients with a typical imaging pattern of UIP especially in the context of rheumatoid arthritis [15]. Overall, diagnosing an underlying CTD impacts the long-term prognosis of patients with ILD as compared to the idiopathic disease, and therefore affects the information delivered to the patient and, potentially, management decisions.

A number of series in the last decade have demonstrated that the histopathological patterns originally individualised in the idiopathic setting can also occur in the context of CTD with varying frequencies. The pattern of NSIP is generally the most frequently observed in patients with CTD-ILD, with the notable exception of a frequent UIP pattern in rheumatoid arthritis $[3,10-13,15]$. A pattern of organising pneumonia (occasionally overlapping with features of NSIP, especially in patients with idiopathic inflammatory myopathy) is also frequent. However, it is now agreed by most experts that a lung biopsy is generally not needed in patients with CTD, especially systemic sclerosis and rheumatoid arthritis. Indeed, the pathologic pattern observed in CTD does not seem to affect the prognosis and survival as clearly as in the idiopathic setting. Although patients with idiopathic NSIP [20] or idiopathic desquamative interstitial pneumonia [21] have a dramatically better long-term survival than those with IPF [22-24], such a difference was not found in patients with systemic sclerosis or rheumatoid arthritis $[3,10]$. Studies correlating the pathological pattern with outcome all suffer the limitation of a high risk of constitutive selection bias, with lung biopsy being performed in individuals with the least typical pattern of disease at imaging. Furthermore, results of the lung biopsy generally do not impact on treatment decisions in the setting of CTD, as corticosteroids and/or immunosuppressive therapy are considered in most patients with progressive and disabling CTD-ILD in the absence of contra-indications. There are notable exceptions to this general comment, and video-assisted surgical lung biopsy may be considered in CTD patients with rare or unusual ILD features at imaging, in cases in which malignant disease cannot be excluded noninvasively (e.g. lymphoma and/or lymphocytic interstitial pneumonia in patients with Sjögren's syndrome), or when the underlying CTD is very poorly characterised due to limited systemic manifestations especially idiopathic inflammatory myopathy (see later).

Diagnosing CTD may impact on patient management, with more frequent use of corticosteroids and immunosuppressive therapy $[25,26]$, and possibly newer biological agents such as rituximab [27]. Beyond the consequences that diagnosing the underlying CTD may have on managing the ILD, diagnosing a CTD may directly impact on the information delivered to the patient regarding the potential risk of systemic manifestations, with further ensuing consequences on the choice of disease modifying drugs or screening for systemic complications (e.g. screening for pulmonary arterial hypertension in systemic sclerosis).

\section{CTD features may be missed in the diagnostic approach of ILD}

Despite practical consequences on the management of both ILD and systemic disease that diagnosing the underlying CTD may have, studies have shown that this diagnosis may not be made in a proportion of the patients seen by chest physicians, even in prestigious ILD centres. In a retrospective study at the Johns Hopkins Centre (Baltimore, MA, USA), 114 out of 160 consecutive patients referred for ILD had at least one auto-antibody (and no other identified cause of ILD), of whom 34 satisfied international criteria for a welldefined CTD; although half of those already had an established diagnosis of CTD, another half of these patients had not been previously diagnosed with CTD [28]. Possible reasons as to why the diagnosis of CTD may be missed in the setting of ILD can be summarised as follows [29].

The respiratory clinical manifestations related to the ILD may precede the systemic symptoms associated with the underlying CTD. Although exceptional in patients with systemic sclerosis, such chronology of events is relatively frequent in those with inflammatory idiopathic myopathy [30-32]. In one series of ILD associated with anti-synthetase antibodies, respiratory manifestations occurred before the onset of muscle or skin disease in 55\% of the patients [32]. Long-term follow-up of patients with NSIP may lead to the subsequent diagnosis of CTD in a significant proportion of patients [20,33,34], suggesting that subtle extra-thoracic features of systemic disease present at baseline might have been overlooked. 
Identification of the CTD may be difficult when the onset of disease is acute or subacute, as often observed in inflammatory idiopathic myopathy (especially associated with anti-melanoma differentiation associated gene (MDA5) antibodies) [35].

Auto-antibodies present may be rare and not systematically tested in all patients presenting with CTD. For example, anti-PL7 and anti-PL12 t-RNA-synthetase antibodies may be occasionally present and be clinically relevant in patients with apparently "idiopathic" ILD without antinuclear antibodies or anti-Jo1 antibodies at autoimmune biology screening tests [36].

The extra-thoracic manifestations of CTD may be subtle to moderate and easily overlooked, especially when not impairing daily life activities and not fulfilling diagnostic criteria for overt CTD. This condition has been coined with various terminology (which largely overlap yet do not exactly match), including UCTD, undifferentiated connective tissue syndromes, early CTD, "formes frustes" of CTD, occult CTD, overlap CTD, lung-limited CTD and autoimmune-featured ILD.

Referral to a rheumatologist may contribute to the appropriate identification of systemic features in patients with suspected CTD or UCTD. Some criteria have been suggested by authors to help identify patients who may be referred to a rheumatologist (table 2) [37]. Multidisciplinary discussion involving pulmonologists and rheumatologists may significantly contribute to improving management.

\section{Are auto-antibody and/or limited extra-thoracic manifestations significant?}

In a landmark study, KINDER et al. [5] evaluated the proportion of patients with "idiopathic" interstitial pneumonia who fit the diagnosis of UCTD using a predefined definition that included the presence of at least one symptom suggestive of CTD and biological evidence of systemic inflammation (corresponding to the presence of auto-antibodies, elevated sedimentation rate or C-reactive protein level). Although some of the clinical and biological features used in this study lack specificity for CTD, the diagnosis of UCTD was made in $62 \%$ of patients. The majority of patients $(71 \%)$ classified as NSIP at lung biopsy met the criteria for UCTD. Furthermore, as compared to the control group, patients with UCTD were more likely: to be female, to be younger, to have ground-glass opacity on HRCT, and to have a NSIP pattern at lung biopsy [5, 38].

Therefore, these findings suggested that subtle clinical and biological features (however not fulfilling diagnostic criteria of overt CTD) were associated with phenotypic changes in patients with ILD. Of note, criteria used in this and other studies conducted by pulmonologists did not always include the criterion of disease duration. Indeed, as systemic manifestations may appear over a period of several months, rheumatologists generally consider the diagnosis of UCTD only after sufficient disease duration has occurred. According to the latter definition $[39,40]$, patients are diagnosed with UCTD in the presence of the following: 1) signs and symptoms suggestive of CTD, but not satisfying the criteria of any defined disease entity; 2) positive results for antinuclear antibody test; and 3) disease duration of at least 3 years.

TABLE 2 Indicative criteria for which patients may be referred for rheumatologic evaluation

\section{Features suggesting CTD}

Demographics

Clinical manifestations

Lung histopathology

Biology

\section{Female sex}

Age $<50$ years

Any patient with extra-thoracic manifestations highly suggestive of CTD: Raynaud's phenomenon, oesophageal hypomotility, inflammatory arthritis of the metacarpophalangeal joints or wrists, digital oedema, symptomatic keratoconjonctivitis sicca

All cases of NSIP, LIP or ILD patterns with histopathology features that might suggest CTD:

Extensive pleuritis, dense perivascular collagen, lymphoid aggregates with germinal centre formation, prominent plasmacytic infiltration

Patients with positive high-titre antinuclear antibodies $(>1: 320)$ or rheumatoid factor $\left(>60 \mathrm{IU} \cdot \mathrm{mL}^{-1}\right)$, nucleolar staining antinuclear antibodies or specific antibodies lanti-cyclic citrullinated peptide, anti-Scl70, anti-Ro, anti-La, anti-dsDNA, anti-Smith, anti-RNP, anti-tRNA synthetasel 
In another study, VIJ et al. [41] prospectively analysed the clinical characteristics, imaging features and outcome of patients with so-called autoimmune-featured ILD, as defined by the presence of at least one symptom of CTD (some of which with low specificity) and one positive serology test using predefined criteria. In this study of 200 patients with ILD, the proportion of males was $59 \%$ in patients with autoimmune-featured ILD, as compared to $74 \%$ of IPF patients and only $24 \%$ of those with ILD in overt CTD $(p<0.01)$ [41]. Patients with the nonspecific criteria of elevated erythrocyte sedimentation rate or Creactive protein were not included in the group of autoimmune-featured ILD in this study. The male to female ratio in autoimmune featured-ILD was $\approx 60: 40$, which was different from IPF $(75: 25)$ and CTD-ILD (25:75). Patients with autoimmune-featured ILD were younger than those with IPF and older than those with CTD-ILD $(\mathrm{p}<0.01)$. Similarly, the proportion of patients with a typical UIP pattern at imaging was $62 \%$ in patients with autoimmune-featured ILD, as compared to $90 \%$ of IPF patients and only $38 \%$ of those with ILD in overt CTD $(\mathrm{p}<0.01)$; however, no difference was found in the severity of pulmonary function impairment [41]. Although the survivors were followed for a median of 1.7 years, it could not be excluded that a subset of this patient population may eventually meet the criteria for a well-defined CTD. These findings demonstrate that identification of subtle CTD clinical features, when associated with presence of auto-antibodies, do impact on the clinical and imaging presentation of ILD and are associated with distinct attributes. In other words, subtle manifestations of CTD should be considered in a patient evaluated for ILD, even if overt CTD criteria are not fulfilled.

In another study, CORTE et al. [6] elegantly demonstrated that the proportion of patients with idiopathic interstitial pneumonia that fulfil UCTD criteria is dependent on the criteria used (table 3). Using "broad criteria" [5], UCTD could be diagnosed in 36\% of patients with IPF and in $71 \%$ of patients with NSIP, whereas UCTD was considered to be present in only $13 \%$ and $21 \%$, respectively, of the same patients groups using the more stringent criteria of UCTD [40]. The presence of UCTD was associated with a higher frequency of NSIP histopathology [6].

In a recent study, FISCHER et al. [17] suggested the terminology of lung-dominant CTD to account for the subset of patients with interstitial pneumonia who have clinical features suggesting an associated CTD, but the features fall short of a clear diagnosis of CTD under the current rheumatologic classification systems. The proposed provisional criteria for this condition include four criteria: 1) a histological or radiological pattern of NSIP, UIP, lymphocytic interstitial pneumonia, organising pneumonia or diffuse alveolar damage; 2) insufficient extra-thoracic features of a definite CTD to allow a specific CTD designation; 3 ) no identifiable alternative aetiology for interstitial pneumonia; 4) any one of a list of auto-antibodies or at least two of four predefined histopathology features (lymphoid aggregates with germinal centres, extensive pleuritis, prominent plasmacytic infiltration and dense perivascular collagen). Such provisional conceptual classification carries the advantage of setting the stage for future studies of the prognostic, therapeutic and pathobiological implications of CTD features in patients with ILD. However, the proposed criteria need further refinement based on evidence, especially since they imply that patients may be classified as having

TABLE 3 Diagnostic criteria for undifferentiated connective tissue disease (UCTD)

UCTD diagnostic criteria ${ }^{\#}$

At least one of: 1) Raynaud's phenomenon;

2) arthralgias/multiple joint swelling; 3) morning stiffness; 4) dry mouth or dry eyes (Sicca features); 5) proximal muscle weakness

\section{B. Positive autoimmune serology}

Positive finding of at least one of: 1) ANA (high titre); 2) RF (high titre); 3) positive ENA; 4) anti-Scl70 antibody; 5) anti-RNP antibody; 6) anticentromere antibody; 7) SS-A or SS-B;

\section{Broader definition of UCTD",}

At least one of: 1) Raynaud's phenomenon; 2) arthralgias/multiple joint swelling; 3) photosensitivity; 4) unintentional weight loss; 5) morning stiffness; 6) dry mouth or dry eyes (Sicca features); 7) dysphagia; 8) recurrent unexplained fever; 9) gastro-oesophageal reflux; 10) skin changes (rash); 11) oral ulceration; 12) nonandrogenic alopecia; 13) proximal muscle weakness Positive finding of at least one of: 1) ANA; 2l RF;

3) anti-Scl70 antibody; 4) SS-A or SS-B; 5) Jo-1 antibody; 6) ESR ( $>2$ times normal), CRP

8) Jo-1 antibody

CTD: connective tissue disease; ANA: antinuclear antibody; RF: rheumatoid factor; ENA: extractable nuclear antigen; RNP: ribonucleoprotein; SS-A: anti-Ro; SS-B: anti-La; ESR: erythrocyte sedimentation rate; CRP: C-reactive protein. * a diagnosis of UCTD requires having one or more of the symptoms outlined in A, and one or more of the laboratory findings listed in B; ": criteria proposed by KINDER et al. [5]. Reproduced from [6] with permission. 
lung-dominant CTD despite the absence of any extra-thoracic features of CTD, and since histopathological features used in this working classification have not been robustly validated.

In a similar approach, several studies have evaluated the potential relevance of evaluating organ-specific manifestations of CTD. In a group of 74 patients with respiratory symptoms and the presence of anti-cyclic citrullinated peptide antibodies (the majority of whom with isolated airways disease, some with isolated ILD or mixed airways disease and ILD including the syndrome of combined pulmonary fibrosis and emphysema), three developed articular manifestations of rheumatoid arthritis during a median follow-up of 449 days [42]. In patients with ILD who were apparently idiopathic (and with no definable CTD), occult Sjögren's syndrome was detected by systematic salivary gland biopsy in 34\% of cases, including $62 \%$ with presence of anti-SSa antibodies and 77\% with sicca symptoms [43]. The concept of UCTD or lungdominant CTD can also apply to patients with limited manifestations of idiopathic inflammatory myopathy (amyopathic dermatomyositis) [12, 44, 45] or systemic sclerosis (fig. 1) [46, 47].

However, limitation to the concept of UCTD-ILD or lung-limited CTD has come from the lack of demonstration of clear impact on long-term survival. Although it is clear that patients with CTD-ILD have improved survival compared to those with $\operatorname{IPF}[3,4,7]$, studies have generally not demonstrated a survival advantage in patients with UCTD-ILD as compared to those with no CTD features [6]. In the study by VIJ et al. [41], the 5-year overall survival of patients diagnosed with UCTD-ILD (52\%) did not differ from that of patients with IPF (48\%), and was significantly lower than that of subjects with CTD-ILD (95\%, p $<0.01)$. Interestingly, antinuclear antibodies when present at a high titre (e.g. $\geqslant 1: 1280$, found in $17(27 \%)$ out of 63 patients) were associated with improved survival in patients with autoimmune featured-ILD $(\mathrm{p}<0.02)$ [41]. This suggests that biological autoimmune features strongly associated with CTD might portend a distinct outcome and possibly improved survival as compared to those with IPF.

Furthermore, the presence of autoantibodies per se is not associated with a survival advantage in patients with IPF and no other features of CTD, and especially in patients with no clinical extra-thoracic manifestation. As compared to IPF patients with negative autoimmune biology, those with auto-antibodies are more likely to exhibit ground-glass attenuation, have more significant honeycombing and a higher extent of ILD at HRCT but similar survival [7]. Similarly, LEE et al. [48] tested 67 patients with IPF and 52 healthy controls for the presence of an extensive panel of auto-antibodies and found no difference in the frequency or type of antibodies between IPF and control subjects (22\% versus $21 \%$ ), probably reflecting auto-antibodies of unknown significance in an ageing population rather than genuine UCTD (although long-term follow-up was not available). Among patients with IPF, no differences were found in clinical, functional or imaging characteristics between those with and without auto-antibodies. When adjusted for age, sex, smoking and forced vital capacity, circulating antibodies (antinuclear antibodies with titre $\geqslant 1: 320$ or rheumatoid factor $\geqslant 60 \mathrm{UI} \cdot \mathrm{mL}^{-1}$ ) seem to be associated with longer transplant-free survival time [48].

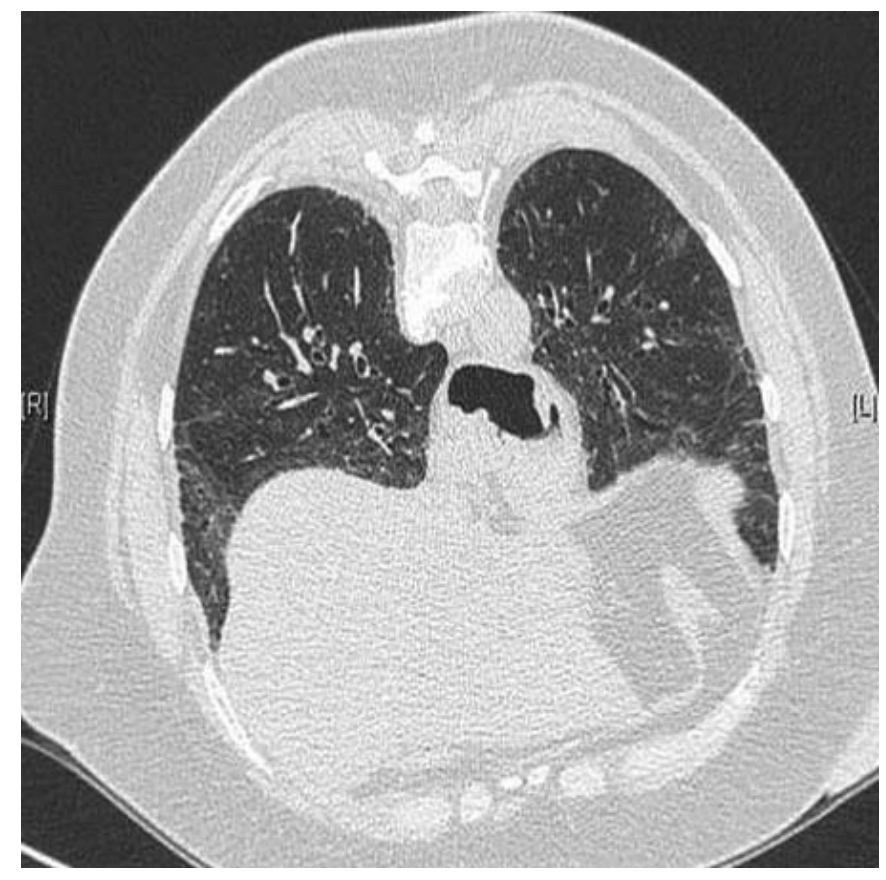

FIGURE 1 High-resolution computed tomography scan of a 59-year-old female patient with undifferentiated connective tissue disease, showing patterns of nonspecific interstitial pneumonia (with ground-glass opacification and traction bronchiectasis) and oesophageal dilation. Features of systemic sclerosis were present (Raynaud's phenomenon, megacapillaries at nailfold capillaroscopy, oesophageal hypomotility, anti-topoisomerase-1 antibodies), but no sclerodactily or telangiectasia was visible at 5 years follow-up. 
Overall, these observations suggest that the frequency of circulating autoantibodies in patients with IPF is similar to that of healthy controls, with unclear impact on survival.

\section{Conclusions}

CTD should be considered in every patient with ILD, particularly females and subjects $<50$ years of age. Such systematic approach is especially justified by the observation that ILD may be the presenting manifestation of CTD and/or the dominant manifestation of CTD. ILD with overt CTD has a better survival than IPF.

Patients with clinical features of CTD and/or auto-antibodies but who do not fit with established criteria for the diagnosis of CTD are being increasingly recognised. These may be called undifferentiated CTD or "lungdominant CTD", and should be considered in the presence of specific auto-antibodies and/or extra-thoracic manifestations. Although the terminology and criteria need further refinement, several studies have showed that when present, limited CTD features may impact on symptoms, imaging features, pathological features and evolution to overt CTD. However, auto-antibodies alone, when present in IPF patients, do not impact on prognosis or management, and it is unclear whether they are associated with distinct survival. Patients with ILD should be considered to be classified as CTD if at least one autoantibody is found (especially with a high titre and/or it is an antibody considered highly specific of an autoimmune condition), and at least one clinical feature other than ILD and typical of CTD. Referral to a rheumatologist and multidisciplinary discussion may contribute to the management of these patients.

\section{References}

1 Fischer A, du Bois R. Interstitial lung disease in connective tissue disorders. Lancet 2012; 380: 689-698.

2 Antin-Ozerkis D, Rubinowitz A, Evans J, et al. Interstitial lung disease in the connective tissue diseases. Clin Chest Med 2012; 33: 123-149.

3 Park JH, Kim DS, Park IN, et al. Prognosis of fibrotic interstitial pneumonia: idiopathic versus collagen vascular disease-related subtypes. Am J Respir Crit Care Med 2007; 175: 705-711.

4 Navaratnam V, Ali N, Smith CJ, et al. Does the presence of connective tissue disease modify survival in patients with pulmonary fibrosis? Respir Med 2011; 105: 1925-1930.

5 Kinder BW, Collard HR, Koth L, et al. Idiopathic nonspecific interstitial pneumonia: lung manifestation of undifferentiated connective tissue disease? Am J Respir Crit Care Med 2007; 176: 691-697.

6 Corte TJ, Copley SJ, Desai SR, et al. Significance of connective tissue disease features in idiopathic interstitial pneumonia. Eur Respir J 2012; 39: 661-668.

7 Song JW, Do KH, Kim MY, et al. Pathologic and radiologic differences between idiopathic and collagen vascular disease-related usual interstitial pneumonia. Chest 2009; 136: 23-30.

8 Le Goff B, Cherin P, Cantagrel A, et al. Pneumomediastinum in interstitial lung disease associated with dermatomyositis and polymyositis. Arthritis Rheum 2009; 61: 108-118.

9 Cottin V, Nunes H, Mouthon L, et al. Combined pulmonary fibrosis and emphysema syndrome in connective tissue disease. Arthritis Rheum 2011; 63: 295-304.

10 Bouros D, Wells AU, Nicholson AG, et al. Histopathologic subsets of fibrosing alveolitis in patients with systemic sclerosis and their relationship to outcome. Am J Respir Crit Care Med 2002; 165: 1581-1586.

11 Kim DS. Interstitial lung disease in rheumatoid arthritis: recent advances. Curr Opin Pulm Med 2006; 12: 346-353.

12 Cottin V, Thivolet-Bejui F, Reynaud-Gaubert M, et al. Interstitial lung disease in amyopathic dermatomyositis, dermatomyositis and polymyositis. Eur Respir J 2003; 22: 245-250.

13 Douglas WW, Tazelaar HD, Hartman TE, et al. Polymyositis-dermatomyositis-associated interstitial lung disease. Am J Respir Crit Care Med 2001; 164: 1182-1185.

14 Tansey D, Wells AU, Colby TV, et al. Variations in histological patterns of interstitial pneumonia between connective tissue disorders and their relationship to prognosis. Histopathology 2004; 44: 585-596.

15 Kim EJ, Elicker BM, Maldonado F, et al. Usual interstitial pneumonia in rheumatoid arthritis-associated interstitial lung disease. Eur Respir J 2010; 35: 1322-1328.

16 Cottin V. Pragmatic prognostic approach of rheumatoid arthritis-associated interstitial lung disease. Eur Respir J 2010; 35: 1206-1208.

17 Fischer A, West SG, Swigris JJ, et al. Connective tissue disease-associated interstitial lung disease: a call for clarification. Chest 2010; 138: 251-256.

18 Devouassoux G, Cottin V, Liote H, et al. Characterisation of severe obliterative bronchiolitis in rheumatoid arthritis. Eur Respir J 2009; 33: 1053-1061.

19 Rajasekaran A, Shovlin D, Saravanan V, et al. Interstitial lung disease in patients with rheumatoid arthritis: comparison with cryptogenic fibrosing alveolitis over 5 years. J Rheumatol 2006; 33: 1250-1253.

20 Romagnoli M, Nannini C, Piciucchi S, et al. Idiopathic nonspecific interstitial pneumonia: an interstitial lung disease associated with autoimmune disorders? Eur Respir J 2011; 38: 384-391.

21 Godbert B, Wissler M-P, Vignaud JM. Desquamative interstitial pneumonia: an analytic review with emphasis on aetiology. Eur Respir Rev 2013; 22: 117-123.

22 Bjoraker JA, Ryu JH, Edwin MK, et al. Prognostic significance of histopathologic subsets in idiopathic pulmonary fibrosis. Am J Respir Crit Care Med 1998; 157: 199-203.

23 Flaherty KR, Mumford JA, Murray S, et al. Prognostic implications of physiologic and radiographic changes in idiopathic interstitial pneumonia. Am J Respir Crit Care Med 2003; 168: 543-548.

24 Flaherty KR, Toews GB, Travis WD, et al. Clinical significance of histological classification of idiopathic interstitial pneumonia. Eur Respir J 2002; 19: 275-283. 

disease-associated interstitial lung disease. J Rheumatol 2013; 40: 640-646.

Tashin DP, Eloff Med 2006; 354: 2655-2666.

27 Keir GJ, Maher TM, Hansell DM, et al. Severe interstitial lung disease in connective tissue disease: rituximab as rescue therapy. Eur Respir J 2012; 40: 641-648.

28 Mittoo S, Gelber AC, Christopher-Stine L, et al. Ascertainment of collagen vascular disease in patients presenting with interstitial lung disease. Respir Med 2009; 103: 1152-1158.

29 Cottin V. Interstitial lung disease: are we missing formes frustes of connective tissue disease? Eur Respir J 2006; 28: 893-896.

30 Matsushita T, Hasegawa M, Fujimoto M, et al. Clinical evaluation of anti-aminoacyl tRNA synthetase antibodies in Japanese patients with dermatomyositis. J Rheumatol 2007; 34: 1012-1018.

31 Yoshifuji H, Fujii T, Kobayashi S, et al. Anti-aminoacyl-tRNA synthetase antibodies in clinical course prediction of interstitial lung disease complicated with idiopathic inflammatory myopathies. Autoimmunity 2006; 39: 233-241.

32 Tillie-Leblond I, Wislez M, Valeyre D, et al. Interstitial lung disease and anti-Jo-1 antibodies: difference between acute and gradual onset. Thorax 2008; 63: 53-59.

33 Homma Y, Ohtsuka Y, Tanimura K, et al. Can interstitial pneumonia as the sole presentation of collagen vascular diseases be differentiated from idiopathic interstitial pneumonia? Respiration 1995; 62: 248-251.

34 Park IN, Jegal Y, Kim DS, et al. Clinical course and lung function change of idiopathic nonspecific interstitial pneumonia. Eur Respir J 2009; 33: 68-76.

35 Koga T, Fujikawa K, Horai Y, et al. The diagnostic utility of anti-melanoma differentiation-associated gene 5 antibody testing for predicting the prognosis of Japanese patients with DM. Rheumatology (Oxford) 2012; 51: $1278-1284$.

36 Fischer A, Swigris JJ, du Bois RM, et al. Anti-synthetase syndrome in ANA and anti-Jo-1 negative patients presenting with idiopathic interstitial pneumonia. Respir Med 2009; 103: 1719-1724.

37 Fischer A, du Bois RM. A practical approach to connective tissue disease-associated lung disease. In: Baughman RM, du Bois RM, eds. Diffuse Lung Disease: A Practical Approach. New York, Springer Science, 2012 ; pp. $217-237$.

38 Kim DS, Nagai S. Idiopathic nonspecific interstitial pneumonia: an unrecognized autoimmune disease? Am J Respir Crit Care Med 2007; 176: 632-633.

39 Doria A, Mosca M, Gambari PF, et al. Defining unclassifiable connective tissue diseases: incomplete, undifferentiated, or both? J Rheumatol 2005; 32: 213-215.

40 Mosca M, Neri R, Bencivelli W, et al. Undifferentiated connective tissue disease: analysis of 83 patients with a minimum follow up of 5 years. J Rheumatol 2002; 29: 2345-2349.

41 Vij R, Noth I, Strek ME. Autoimmune-featured interstitial lung disease: a distinct entity. Chest 2011; 140: 1292-1299.

42 Fischer A, Solomon JJ, du Bois RM, et al. Lung disease with anti-CCP antibodies but not rheumatoid arthritis or connective tissue disease. Respir Med 2012; 106: 1040-1047.

43 Fischer A, Swigris JJ, du Bois RM, et al. Minor salivary gland biopsy to detect primary Sjogren syndrome in patients with interstitial lung disease. Chest 2009; 136: 1072-1078.

44 Euwer RL, Sontheimer RD. Amyopathic dermatomyositis (dermatomyositis sine myositis). Presentation of six new cases and review of the literature. I Am Acad Dermatol 1991; 24: 959-966.

45 Euwer RL, Sontheimer RD. Amyopathic dermatomyositis: a review. J Invest Dermatol 1993; 100: 124S-127S.

46 Poormoghim H, Lucas M, Fertig N, et al. Systemic sclerosis sine scleroderma: demographic, clinical, and serologic features and survival in forty-eight patients. Arthritis Rheum 2000; 43: 444-451.

47 Lomeo RM, Cornella RJ, Schabel SI, et al. Progressive systemic sclerosis sine scleroderma presenting as pulmonary interstitial fibrosis. Am J Med 1989; 87: 525-527.

48 Lee JS, Kim EJ, Lynch KL, et al. Prevalence and clinical significance of circulating autoantibodies in idiopathic pulmonary fibrosis. Respir Med 2013; 107: 249-255. 